\title{
O uso do correio eletrônico na comunicação entre usuários e uma equipe de saúde da família: relato de experiência
}

\author{
The use of electronic mail between users and a primary health care team: a case report
}

\section{El uso del correo electronico en la comunicación entre usuarios y un equipo de atención primaria: relato de experiencia}

Paulo Poli Neto. Universidade Federal do Paraná (UFPR). Curitiba, PR, Brasil. ppolineto @ gmail.com (Autor correspondente)

Charles Dalcanale Tesser. Universidade Federal de Santa Catarina (UFSC). Florianópolis, SC, Brasil. charles.tesser@ufsc.br Gabriel Hahn Monteiro. Centro de Pesquisas em Oncologia (CEPON). Florianópolis, SC, Brasil. gabriel.lufchitz@gmail.com Pedro Felipe Mylla Boso. Hospital Governador Celso Ramos, Florianópolis, SC, Brasil. pedro_boso@ hotmail.com George Lemos. Hospital Nossa Senhora das Graças, Curitiba, PR, Brasil. grlemos@ hotmail.com

\section{Resumo}

Este relato descreve o uso do correio eletrônico por uma equipe de saúde da família para comunicação com os pacientes e agendamento. Tal uso surgiu como consequência de um processo de facilitação do acesso presencial e não presencial. No período de 25 meses houve crescimento no número de mensagens e de remetentes. Das 1437 mensagens recebidas, $61,5 \%$ foram para agendar consultas, $27,8 \%$ solicitavam informações em saúde e $7,6 \%$ eram sobre serviços oferecidos. As mulheres enviaram $87 \%$ das mensagens ( $63,3 \%$ delas tinham $20-40$ anos). Em $60 \%$ das vezes, o contato referia-se à demanda do remetente e mais de $30 \%$ à de familiares. A equipe respondeu a $83 \%$ das mensagens em até 8 horas úteis. Na percepção da equipe, o uso do correio eletrônico melhorou a comunicação e o acesso e foi bem aceito pelos usuários, não prejudicando grupos mais vulneráveis. Investigações mais aprofundadas serão necessárias para avaliar a contribuição dessa ferramenta na Atenção Primária à Saúde.

\section{Abstract}

This report describes the use of electronic mail by a primary health care team (PHT) in order to communicate with patients and aid in scheduling. Such use has emerged as a result of a process of facilitating access, both electronically and face-to-face. During a 25-month study period, users sent a total of 1437 messages with the purpose of scheduling appointments $(61.5 \%)$, asking health-related questions (27.8\%), and inquiring about services offered $(7.6 \%)$. Women submitted $87 \%$ of the messages ( $63.3 \%$ of them were $20-40$ years of age), with $60 \%$ of these messages concerning themselves and over $30 \%$ of them concerning their families. The team responded to $83 \%$ of messages within 8 business hours. According to the team, the use of electronic mail has improved communication and access, and has been well received by users without harming the most vulnerable groups. Further investigation is needed to assess the full contribution of this tool to primary health care.

\section{Palavras-chave:}

Correio Eletrônico

Acesso aos Serviços de Saúde

Comunicação

Atenção Primária à Saúde

Keywords:

Eletronic Mail

Health Service Accessibility

Communication

Primary Health Care
Como citar: Poli Neto P, Tesser CD, Monteiro HG, Boso P, Lemos G. O uso do correio eletrônico na comunicação entre usuários e uma equipe de saúde da família: relato de experiência. Rev Bras Med Fam Comunidade. 2015;10(37)1-9. http://dx.doi.org/10.5712/rbmfc10(37)1167
Fonte de financiamento: declaram não haver. Parecer CEP: não se aplica. Conflito de interesses: declaram não haver. Procedência e revisão por pares: revisado por pares. Recebido em: 22/06/2015. Aprovado em: 08/11/2015. 


\section{Resumen}

Este texto describe la utilización del correo electronico por un equipo de salud de la familia para comunicación con los pacientes y marcación de citas. El correo surgió como consecuencia de un proceso para facilitar el acceso presencial y no presencial. En el periodo de 25 meses hubo crecimiento en el numero de mensajes y de remitentes. De las 1.437 mensajes recibidas, el $61,5 \%$ fueron para la marcación de citas, el 27,8\% solicitaban informaciones de salud y el 7,6\% eran sobre servicios ofrecidos. Las mujeres enviaron el $87 \%$ de los correos (el $63,3 \%$ de ellas tenían entre 20 y 40 años). En el $60 \%$ de las veces, el contacto se refería a la demanda del remitente y más del $30 \%$ a las de sus familiares. El equipo contestó al $83 \%$ de los mensajes dentro de 8 horas útiles. Según el equipo, el uso del correo electrónico mejoró la comunicación y el acceso y fue bien acepto por los usuarios, sin perjudicar a los grupos más vulnerables. Investigaciones más profundas serán necesarias para evaluar la contribución de esta herramienta en la Atención Primaria a la Salud.
Palabras clave:

Correo Electrónico

Acceso a los Servicios

de Salud

Comunicación

Atención Primaria de Salud

\section{Introdução}

O acesso é um dos pilares da organização de qualquer rede de serviços de saúde. Em muitos países, cabe às unidades de Atenção Primária (APS), compostas por médicos e enfermeiros generalistas, o papel de ser a porta de entrada e de atenção continuada para a maioria das demandas de uma população definida. ${ }^{1,2}$ Nesse contexto, é importante que a comunicação dos usuários com suas equipes de APS seja facilitada, permitindo consultas presenciais no momento adequado. . $^{3,4}$ Uma possibilidade nesse sentido é a dos contatos não presenciais, como telefone, mensagens de texto, correio eletrônico, dentre outras.

Avaliações de qualidade da APS realizadas a partir dos usuários, como a do Primary Care Assessment - tool (PCA-Tool) ${ }^{5}$ e a enquete realizada regularmente na Grã-Bretanha, avaliam o acesso por meio da facilidade em se conseguir uma consulta em até $48 \mathrm{~h}$, por oferecer ou não assistência fora do horário comercial e, também, pela possibilidade de um contato não-presencial, como por telefone ou e-mail. ${ }^{6}$

Uma característica do contato não presencial é que pode otimizar a utilização do tempo do usuário e da equipe ao evitar deslocamentos desnecessários, possibilitar agendamentos, orientações sobre algumas situações de saúde e questões administrativas. O contato não presencial vem sendo visto como uma ferramenta de comunicação e melhoria do acesso, considerando a disponibilização progressiva de novas tecnologias à população e aos profissionais, com custos cada vez menores.

O agendamento de consultas por telefone é prática bem estabelecida nos países desenvolvidos com APS forte e no Brasil no sistema privado, porém muito pouco utilizado no Sistema Único de Saúde (SUS). A experiência mais conhecida no SUS é a do SAMU 192 (Serviço de Atendimento Móvel de Urgência), que faz a regulação telefônica da utilização de ambulâncias para o atendimento fora do domicílio de situações de urgências e emergências. Em outros países, o uso do telefone para orientações aos usuários na APS é consolidado. ${ }^{7}$ A maioria da população tem acesso, a resposta é imediata ou rápida, mas para os profissionais de saúde pode não ser tão satisfatório por ter o potencial de interromper atendimentos. ${ }^{8}$ No Reino Unido, ${ }^{6} 88,4 \%$ dos usuários agenda sua consulta por telefone. Para $71,8 \%$ deles, é fácil acessar a clínica de APS por esse meio, mas em anos anteriores chegava a 76,6\%. O agendamento pela Internet passou de 3,2\% em 2012 para 5,2\% em $2015 .^{6}$

A comunicação por correio eletrônico é mais recente e menos disseminada. O e-mail traz vantagens para a comunicação usuário-equipe, como a de não interromper as atividades dos profissionais e o de tornar as demandas e as respostas mais objetivas e organizadas. ${ }^{9} \mathrm{O}$ acesso à Internet pelos brasileiros 
vem crescendo, como pode ser constatado pelos últimos censos domiciliares realizados, o que faz do correio eletrônico uma possibilidade concreta de contato com seus profissionais de saúde. ${ }^{10}$

Embora haja polêmica sobre o uso do e-mail na APS, ${ }^{11}$ há consistentes indícios e argumentos a seu favor e recomendações a respeito. Entre as 40 respostas rápidas ao artigo de debate de Gunning e Richards ${ }^{11}$ presentes no site do BMJ (em 27/10/2015), aproximadamente 27 comentários se manifestam favoráveis (ressalvando cuidados com segurança e limites dessa via de comunicação) e 13 contrariamente ao uso do e-mail. Os argumentos contrários são baseados no medo de ser 'inundado' por e-mails, do uso impróprio pelos pacientes, de potenciais problemas de segurança, e de medo (ou recusa ou consideração) de que é inviável o trabalho extra envolvido em responder às mensagens. Os argumentos a favor convergem com os mencionados neste relato e consideram também que é inevitável o uso desses novos meios de comunicação.

O Institute of Medicine, dos Estados Unidos, recomenda o uso da comunicação por e-mail entre usuários e profissionais para a resolução de problemas não urgentes, pelo potencial de abordar inúmeras situações clínicas e administrativas, com otimização do tempo, melhora do cuidado e continuidade do atendimento. ${ }^{12,13}$

O uso do correio eletrônico possibilita a comunicação rápida com o profissional responsável e uma ampla oferta de serviços, como a marcação de consultas, a renovação de receitas, avaliação e exames complementares, troca de dados em formas de textos e imagens e orientações gerais sobre saúde e sobre o funcionamento dos serviços. ${ }^{14}$ Ele pode ser usado também para a divulgação de informações e assuntos relativos à comunidade, visando à difusão de informações como instrumento complementar na educação em saúde. ${ }^{14}$

A comunicação por e-mail e outros meios não presenciais (sobretudo o telefone) tem seu lugar como complementar aos encontros presenciais, para incrementar a longitudinalidade e a diversidade dos modos de comunicação e orientação aos usuários, dentro de esforços para melhoria da adequação das várias respostas aos distintos tipos de demandas presentes na APS, de modo que os encontros presenciais sejam mais dedicados a quem deles mais precisa, e sejam reduzidos quando desnecessários. ${ }^{15}$

Pesquisa inglesa de satisfação mostrou que a maioria dos usuários gostaria de ter acesso ao seu médico por meio do correio eletrônico: $90 \%$ dos entrevistados gostaria deste contato e essa possibilidade determinaria inclusive a escolha por aquele profissional para $56 \%$ deles. ${ }^{16}$

Recentemente, Newhouse et al., ${ }^{17}$ pesquisando 14 países europeus, encontraram que o uso de e-mail é muito variado, indo de $50 \%$ dos usuários na Dinamarca (em que os médicos da APS são obrigados a oferecer contato por e-mail aos usuários ${ }^{18}$ ) até $18 \%$ na França.

Em uma revisão sobre o tema nas principais bibliotecas virtuais, como Scielo, PubMed e Google Scholar, utilizando os descritores de ciências da saúde "correio eletrônico" e "atenção primária" e "comunicação" não foram encontrados estudos brasileiros ou no Brasil sobre o uso da comunicação por e-mail entre profissionais de saúde e seus pacientes regulares. Um estudo apenas relatou a experiência da utilização do correio eletrônico como uma ferramenta de telediagnóstico na área de odontologia no sul do país. ${ }^{19}$

O objetivo deste relato é descrever e analisar a experiência do uso dessa ferramenta entre junho de 2010 e agosto de 2012 por uma equipe de saúde da família. 


\section{Descrição da experiência}

A experiência é apresentada a partir de duas fontes de informação: a) uma narrativa sobre o processo de implantação do correio eletrônico pela equipe. Um dos autores foi o responsável por implementar essa estratégia, operacionalizá-la junto à equipe, divulgar para os usuários e cuidar da conta nesse período; b) apresentação e discussão de alguns dados disponibilizados pela equipe e pela instituição (Secretaria Municipal de Saúde de Florianópolis) sobre a evolução de sua utilização, como o número e objetivo geral das mensagens, tipo de remetente e tempo de resposta dos profissionais. Essas informações foram alimentadas à época pela equipe em uma planilha $E x c e \Gamma^{\mathrm{TM}}$ com o objetivo de monitorar e avaliar a utilização do correio eletrônico.

Quanto aos aspectos éticos, o relato foi construído a partir da narrativa de um dos autores que participaram da experiência. Os eventos relatados ocorreram dentro da rotina institucional regularmente executada pela equipe de saúde da família, não sendo objeto de sigilo ou confidencialidade. Os dados secundários apresentados, disponibilizados pela equipe e pela instituição não envolvem informações confidenciais de usuários ou profissionais, de modo que nenhuma informação sigilosa foi envolvida. Há algum conflito de interesses na medida em que um dos seus autores participou da experiência, o que viabiliza o relato e não o prejudica significativamente, desde que isso seja levado em conta.

A equipe de saúde da família Laranja (ESFL) pertence ao Centro de Saúde (CS) Ingleses, localizado na zona norte do município de Florianópolis-SC, e é uma das suas seis equipes de saúde da família. A população estimada dessa equipe era de 3600 pessoas à época. O bairro sofreu grandes transformações nas últimas décadas, passando de uma vila de pescadores e pequenos agricultores para uma importante zona turística da cidade. O CS é responsável por mais de 24 mil pessoas, grande parte representada por adultos jovens que trabalham na área de serviços.

Por ser uma região afastada do centro da cidade e pela rápida expansão da população, o CS tinha dificuldade para atrair e manter profissionais, especialmente médicos e enfermeiros, o que dificultava melhorias na organização do acesso. Antes da oferta do correio eletrônico, que iniciou com a ESFL e chegou a ser utilizado por outras equipes, o CS reorganizou todo o processo de trabalho nesse período. Cada uma das equipes passou a ter dois consultórios, um para o médico e outro para o enfermeiro, com grande oferta de consultas para o mesmo dia ou dia seguinte. ${ }^{20}$ Duas equipes, dentre elas a ESFL, passaram a utilizar o telefone para agendamento de consultas nas primeiras duas horas da manhã e da tarde.

Essas mudanças foram muito bem aceitas pela população, que passou a utilizar mais o CS como referência para suas principais demandas. A utilização do correio eletrônico surgiu nesse movimento, como uma possibilidade a mais para os usuários agendarem consultas e dialogarem com seu médico ou enfermeiro e se tornou possível graças à facilidade para agendar com as outras modalidades de acesso já oferecidas. O fato de o CS ser informatizado, ter prontuário eletrônico e acesso à Internet foi outra condição essencial.

A ESFL abriu uma conta de e-mail em um dos grandes servidores mundiais, pela facilidade de manuseio. A população foi avisada dessa nova possibilidade de comunicação de diversas maneiras, inicialmente pelos próprios profissionais da equipe (médico, enfermeira, agentes comunitários de saúde [ACS] e técnicos de enfermagem). Mais tarde, confeccionou-se um carimbo com o endereço do e-mail que 
era registrado no cartão de atendimento dos pacientes (com recursos dos próprios profissionais da equipe). Por fim, elaborou-se um cartão de visitas da equipe (também com recursos dos profissionais), com o nome do CS, da equipe, dos profissionais, a organização do acesso e as formas de contato (e-mail e telefone).

Todos os dias, o médico e a enfermeira da ESFL (ambos com carga horária de 40h semanais), disponibilizavam suas agendas das $8 \mathrm{~h}$ às $10 \mathrm{~h}$ e das $13 \mathrm{~h}$ às $15 \mathrm{~h}$ para o atendimento das pessoas que buscavam diretamente o CS ou agendavam por telefone. Um telefone celular ficava com um dos ACS, que se revezavam em uma recepção exclusiva da ESFL para o agendamento presencial e telefônico. A conta de e-mail era acessada durante todo o período de trabalho, pelo médico ou pela enfermeira, e as mensagens eram vistas e respondidas nos intervalos entre as consultas ou no final dos períodos da manhã e da tarde. Quando se tratava de um pedido de agendamento de consulta, respondia-se com a oferta de um horário de acordo com a necessidade ou pedido específico do usuário.

Como o correio eletrônico é gratuito e os computadores eram de uso rotineiro no serviço, não houve custo adicional na sua implantação.

\section{Evolução do uso do correio eletrônico}

A equipe contabilizou todas as mensagens enviadas e recebidas no que diz respeito ao número de e-mails recebidos e ao número total de contatos de usuários diferentes mensalmente no período de junho de 2010 a agosto de 2012. A utilização do e-mail para a comunicação com a equipe cresceu de forma continuada desde sua implantação, em julho de 2010 (Gráfico 1).

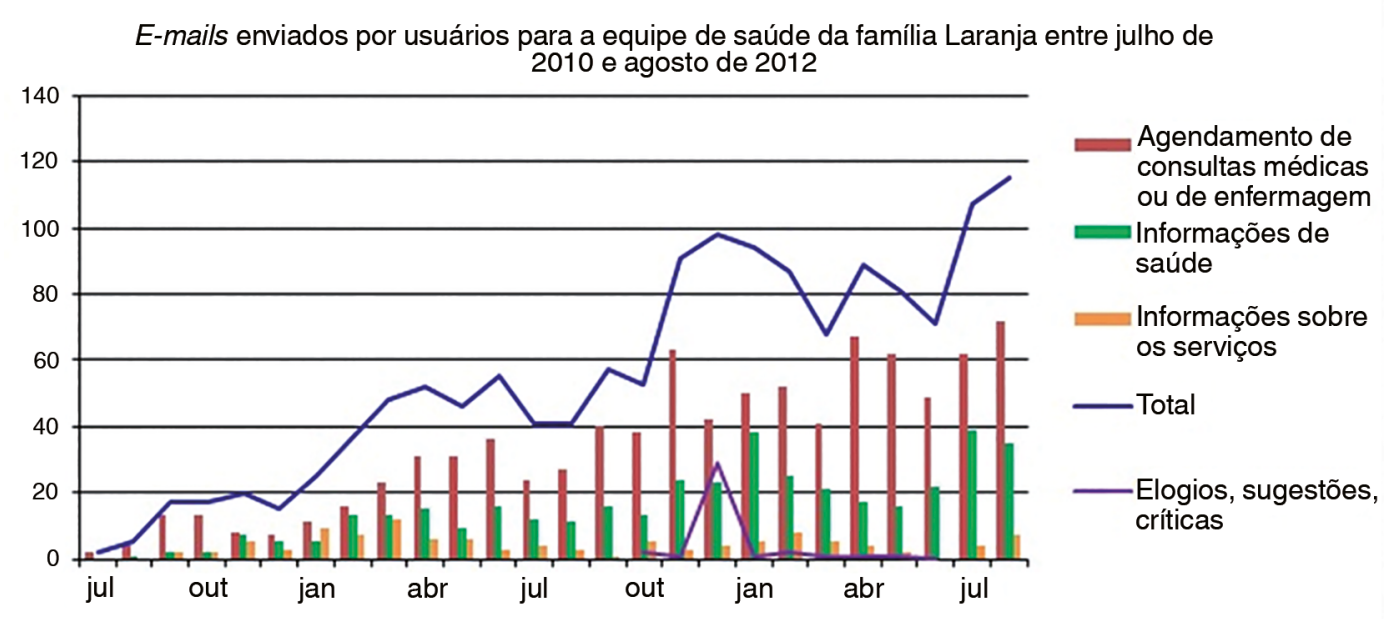

Gráfico 1. E-mails enviados por usuários para a equipe de saúde da família Laranja entre julho de 2010 e agosto de 2012.

Durante esse período, uma planilha $E x c e \Gamma^{\mathrm{TM}}$ foi alimentada com informações sucintas sobre os e-mails enviados pelos usuários, como gênero, data de nascimento, data do envio, motivo da mensagem, tempo de resposta da equipe. O uso do e-mail foi classificado pela equipe em quatro categorias: agendamento de consultas, informações em saúde, informações sobre o serviço e uma quarta com elogios, sugestões e críticas. 
Em $60 \%$ das mensagens, a demanda dos usuários foi o agendamento de consultas. Em seguida, vieram as "informações em saúde" (22\%), que incluíam dúvidas sobre problemas clínicos comuns, orientações gerais sobre intervenções, dietas, atividade física, medicamentos, renovação de receitas, solicitação de exames laboratoriais, entre outros. Na categoria "informações sobre os serviços" surgiam questões sobre os horários e formas de acesso às atividades realizadas no CS, andamento de exames e de encaminhamentos, orientações sobre acesso a outros serviços de saúde e especialidades. No Gráfico 1 vê-se que a proporção entre os dois principais grupos, agendamento e informações de saúde, manteve-se estável, acompanhando o crescimento do total de mensagens.

O aumento no número de mensagens foi provavelmente relacionado também ao aumento no número de contatos (contas de e-mail) diferentes que utilizaram essa ferramenta, que chegou a 404 em agosto de 2012.

A equipe observou que a mesma conta do remetente vinha sendo utilizada para pessoas diferentes em mais de $30 \%$ das vezes, como para os filhos, pais, cônjuge e outros familiares, o que reforça o aspecto familiar e a amplitude da comunicação por e-mail.

Os usuários do e-mail foram $87 \%$ das vezes mulheres, sobretudo jovens e de meia idade $(63,3 \%$ entre 20 e 40 anos; $24,8 \%$ entre 40 e 60 anos; e 10,1\% acima de 60 anos).

O Gráfico 2 mostra o tempo decorrido do momento em que o e-mail foi recebido pela equipe até o momento em que ele foi respondido. Ao se considerar as "horas úteis" (horas dentro do horário de trabalho da equipe de saúde da família) até a resposta ser enviada, a maioria dos e-mails (cerca de 83\%) foi respondida em até 8 "horas úteis", mostrando bastante agilidade nessa comunicação. Segundo relato da equipe, a grande maioria das mensagens foi respondida dentro do horário de trabalho.

Número de e-mails por tempo (em minutos) de resposta da equipe aos usuários entre julho de 2010 e agosto de 2012

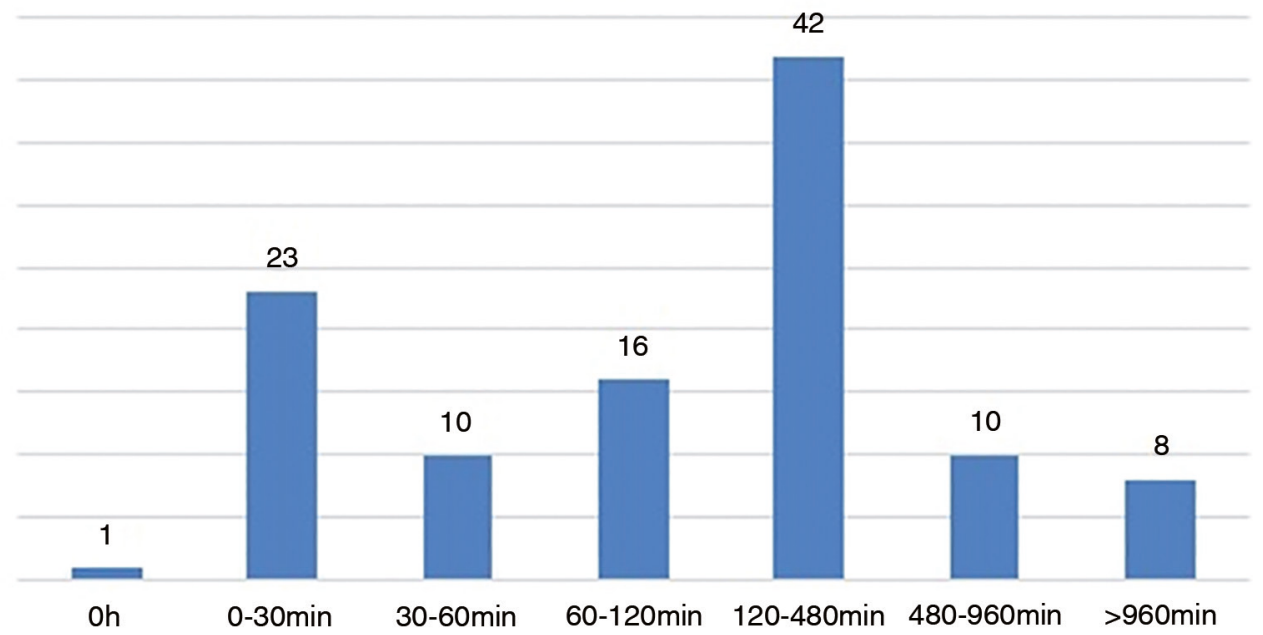

Gráfico 2. Número de e-mails por tempo (em minutos) de resposta da equipe aos usuários entre julho de 2010 e agosto de 2012. 
O tempo gasto na resposta ao e-mail depende de vários fatores, como tamanho da mensagem recebida e complexidade da resposta, agilidade na sua viabilização e agilidade de leitura e digitação. Questões sobre orientações e informações em saúde e sobre serviços do CS provavelmente são respondidas de imediato após a leitura da mensagem, quando o médico ou a enfermeira são os respondentes (com alguma esperada maior autonomia do médico). Solicitações de agendamento dependem da organização e rotina da equipe.

Uma simulação pode ilustrar o processo, proporcionando uma estimativa de tempo gasto nas respostas aos e-mails. Ela pode ser feita a partir da velocidade de digitação, considerando que as mensagens recebidas são em sua grande maioria relativamente curtas e de fácil e rápida leitura. Supondo que o profissional digite em uma velocidade de aproximadamente 200 caracteres por minuto, que o número de mensagens recebidas pela equipe seja de 115 por mês, como mostra o Gráfico 1 após 1,5 anos de uso, e que as respostas sejam relativamente curtas, de até quatro linhas (com 70 caracteres) cada uma, tem-se que o tempo médio para responder à maioria dos e-mails seria em torno de 84 segundos, o que significa pouco mais de 2,5 horas mensais ou 36 minutos por semana nessa atividade. Se acrescentarmos a esse tempo de digitação o tempo necessário para alternância entre softwares, entrada na Internet e acesso ao editor do correio eletrônico, o tempo total parece não ser grande dentro das 40h semanais do médico e do enfermeiro, frente ao benefício potencial.

\section{Discussão}

O uso do e-mail nesta experiência foi na grande maioria das vezes por iniciativa dos usuários. Diferentemente, Car e Sheikh ${ }^{16}$ mencionam que $83 \%$ dos seus e-mails (levantamento de um ano, total de 84 mensagens) eram iniciativa suas ou do serviço, o que faz com que o conteúdo dos e-mails, nesse caso, seja mais administrativo. Estudos realizados em Portugal, uma realidade de APS mais próxima da brasileira, mostraram maior similaridade com este relato, em que há bem maior iniciativa de uso do e-mail pelos usuários, para diversas finalidades, como orientações, esclarecimento de dúvidas, agendamentos e informações administrativas. ${ }^{8,9,14}$

Em pesquisa norte-americana, ${ }^{21}$ constatou-se que de 1 a $10 \%$ dos entrevistados (dependendo da região) têm acesso à comunicação eletrônica com seu médico. Ao responderem sobre a quais serviços gostariam de ter acesso por e-mail, citaram acompanhamento de situações clínicas, informações sobre receitas medicamentosas, envio e análise de exames. Também foi lembrado que o profissional de saúde poderia enviar avisos sobre questões de saúde para a comunidade (como campanhas de vacinação), marcar e desmarcar consultas e renovar prescrições de continuidade.

Quanto à resposta dos e-mails e ao tempo implicado nela, estudos portugueses 9,14 mostraram resultados relativamente semelhantes: também lá, apesar do ritmo acelerado de trabalho, profissionais absorveram o uso do e-mail (com progressivo manuseio dos profissionais administrativos). $\mathrm{O}$ tempo de resposta era semelhante (até $12 \mathrm{~h}$ ou dois dias após o recebimento para a maior parte das mensagens recebidas. ${ }^{14}$ ) Ponte ${ }^{8}$ observou o mesmo tempo médio gasto nas respostas que estimamos, cerca de 30 minutos por semana. Se na APS dos países de alta renda, com menos usuários por equipe, o tempo gasto nas respostas é uma das preocupações, pois significa trabalho extra para os profissionais, no contexto brasileiro, com piores condições socioeconômicas e equipes subdimensionadas, esse tempo é também 
relevante para a viabilidade do uso. Nesta experiência, tal tempo se mostrou aceitável, pelo relato dos profissionais e pela simulação acima. Em todo o caso, o uso do e-mail deve ser considerado no contexto da agilização do acesso e da comunicação dos usuários com a equipe, com esquema ágil de agendamento e uso concomitante de telefone, objetivando diminuir idas desnecessárias dos usuários ao serviço.

Em outros estudos de utilização do e-mail na APS também a maioria dos utilizadores pertence ao sexo feminino ${ }^{9,14}$ (exceção feita ao estudo de Newhouse et al. ${ }^{17}$ ), encontra-se na faixa etária entre 30 e 60 anos e possui escolaridade superior a 8 anos.

Segundo Car e Sheikh, ${ }^{16}$ uma das principais preocupações com a utilização do e-mail é favorecer o acesso a uma parcela da população com mais recursos, reproduzindo "a lei dos cuidados inversos". Esse é um cuidado relevante em situações sociais muito precárias, comuns no Brasil, que deve ser observado. Nesta experiência, tendo isso em vista, o e-mail foi oferecido acompanhado de outras formas de facilitação e agilização de acesso, como a consulta-dia (atendimento com horário agendado para o mesmo dia) marcada por telefone ou presencial e disponibilidade de grande tempo do médico e enfermeira para os demandantes presenciais do dia, facilitando ao máximo o acesso. Na percepção empírica da equipe o uso do e-mail não reforçou a leis dos cuidados inversos. Ao contrário, parece ter tornado o acesso e o agendamento presencial algo mais disponíveis para todos, sem prejuízo dos mais vulneráveis ou com menos recursos, o que, todavia, não se pode afirmar. Novas experiências e estudos adicionais são necessários para avaliação desses aspectos críticos. Também não é possível estimar se o uso do e-mail e do telefone diminuiu ou aumentou o volume de atendimentos presenciais.

\section{Considerações finais}

Supondo a existência de microcomputadores conectados à Internet nos serviços, a implantação do uso do correio eletrônico é relativamente simples se considerarmos que o custo é praticamente nulo e só exige mobilização da equipe, disciplina e disponibilidade dos profissionais que o manuseiam e divulgação aos usuários. Após 25 meses de utilização do e-mail na comunicação entre a equipe de saúde da família e a população vinculada, houve um crescimento constante de seu uso, no número de mensagens e no número de contatos diferentes. Variados usos ocorreram, como marcação de consultas, troca de informações e esclarecimento de dúvidas entre a equipe de saúde e sua população, com adesão significativa dos usuários. Nesta experiência, o tempo de trabalho envolvido nas respostas foi relativamente pequeno e aceitável.

O serviço de e-mail proporciona benefícios à APS, como a não restrição da interação ao encontro presencial, a diminuição de tempo de resposta ao paciente, a facilidade na apresentação de exames ou dúvidas e a possibilidade de informações e orientações de saúde, sendo especialmente útil no acompanhamento de usuários com problemas crônicos.

Parece haver espaço para o crescimento do uso do e-mail na APS brasileira, devido à propagação da Internet entre os usuários. Sugere-se novas experiências e pesquisas com o objetivo de avaliar as preferências dos pacientes e dos profissionais, a melhor maneira de integrar o e-mail aos serviços, a possível influência desse recurso na relação equipe ou médico-usuários e a identificação de possíveis grupos sociais mais beneficiados. Com isso, poderão ser identificadas as adaptações, condições e cuidados necessários para não haver reforço da lei dos cuidados inversos com o uso do correio eletrônico na APS do país. 


\section{Referências}

1. Dixon S, Sampson FC, O'Cathain A, Pickin M. Advanced access: more than just GP waiting times? Fam Pract. 2006;23(2):233-9. DOI: http://dx.doi.org/10.1093/fampra/cmi104

2. Starfield B. Atenção Primária: equilíbrio entre necessidades de saúde, serviços e tecnologia. UNESCO, Ministério da Saúde: Brasília; 2002 [acesso 12 Jun 2015]. Disponível em: http://bvsms.saude.gov.br/bvs/publicacoes/atencao_primaria_p1.pdf

3. Murray M, Berwick DM. Advanced access: reducing waiting and delays in primary care. JAMA. 2003;289(8):1035-40. DOI: http://dx.doi.org/10.1001/jama.289.8.1035

4. Schoen C, Osborn R, Doty MM, Bishop M, Peugh J, Murukutla N. Toward higher-performance health systems: adults' health care experiences in seven countries, 2007. Health Aff (Millwood). 2007;26(6):w717-34. DOI: http://dx.doi.org/10.1377/ hlthaff.26.6.w717

5. Harzheim E, Oliveira MMC, Agostinho MR, Hauser L, Stein AT, Gonçalves MR, et al. Validação do instrumento de avaliação da atenção primária à saúde: PCATool-Brasil adultos. Rev Bras Med Fam Comunidade. 2013;8(29):274-84. DOI: http://dx.doi.org/10.5712/rbmfc8(29)829

6. NHS - The GP Patient Survey - National Sumary Report (January 2015 Publication) [acesso 11 Jun 2015]. Disponível em: http://gp-survey-production.s3.amazonaws.com/archive/2015/January/January\%202015\%20National\%20Summary\%20 Report.pdf

7. Bunn F, Byrne G, Kendall S. Telephone consultation and triage: effects on health care use and patient satisfaction. Cochrane Database Syst Rev. 2004;(4):CD004180.

8. Ponte C. A utilização do correio electrónico na comunicação com os utentes da USF Porta do Sol. Rev Port Clin Geral. 2011;27(3):274-80.

9. Pinhão R, Calisto M, Pimentel MA, Fernandes R. Implementação da comunicação médico-doente via endereço electrónico na consulta de MGF. Rev Port Clin Geral. 2009;25(6):634-8.

10. Programa Nacional de Banda Larga (PNBL) - Brasil Conectado. Presidência da República Federativa do Brasil, 2010 [acesso 15 Jun 2015]. Disponível em: http://www.mc.gov.br/publicacoes

11. Gunning E, Richards E. Should pacients be able to e-mail theirs general practitioner? BMJ 2014;349:g5338. DOI: http:// dx.doi.org/10.1136/bmj.g5338

12. Committee on Quality Health Care, Institute of Medicine. Crossing the Quality Chasm: a new health system for the $21 \mathrm{st}$ century. Washington, DC: National Academy Press; 2001 [acesso 11 Jun 2015]. Disponível em: http://books.nap.edu/html/ quality_chasm/reportbrief.pdf

13. Patt MR, Houston TK, Jenckes MW, Sands DZ, Ford DE. Doctors who are using e-mail with their patients: a qualitative exploration. J Med Internet Res. 2003;5(2):e9. DOI: http://dx.doi.org/10.2196/jmir.5.2.e9

14. Monica G. O uso do e-mail na comunicação com o médico de família: catorze meses de experiência. Rev Port Clin Geral 2009;25(6):639-46.

15. Gérvas J, Bonis J. El debate profesional acerca de la escasez de médicos. Rev Esp Salud Pública 2008; 82(6):627-35.

16. Car J, Sheikh A. Email consultations in health care: 2--acceptability and safe application. BMJ. 2004;329(7463):439-42. DOI: http://dx.doi.org/10.1136/bmj.329.7463.439

17. Newhouse N, Lupiáñez-Villanueva F, Codagnone $\mathrm{C}$, Atherton H. Patient use of email for health care communication purposes across 14 European countries: an analysis of users according to demographic and health-related factors. J Med Internet Res. 2015;17(3):e58. DOI: http://dx.doi.org/10.2196/jmir.3700

18. Danish Ministry of Health. eHealth in Denmark: eHealth as a part of a coherent Danish health care system. 2012 [acesso 11 Jun 2015]. Disponível em: URL: http://www.sum.dk/ /media/Filer\%20-\%20Publikationer_i_pdf/2012/Sundheds-IT/ Sundheds_IT_juni_web.ashx

19. Torres-Pereira C, Possebon RS, Simões A, Bortoluzzi MC, Leão JC, Giovanini AF, et al. Email for distance diagnosis of oral diseases: a preliminary study of teledentistry. J Telemed Telecare. 2008;14(8):435-8. DOI: http://dx.doi.org/10.1258/ jtt.2008.080510

20. Gusso G, Poli Neto P. Gestão da Clínica. In: Gusso G, Ceratti JM Lopes. Org. Tratado de Medicina de Família e Comunidade. Princípios, formação e prática. $1^{\underline{a}}$ ed. Porto Alegre: Artmed; 2012. p.161-5.

21. Houston TK, Sands DZ, Jenckes MW, Ford DE. Experiences of patients who were early adopters of electronic communication with their physician: satisfaction, benefits, and concerns. Am J Manag Care. 2004;10(9):601-8. 